\title{
Chemical Profile and Pancreatic Lipase Inhibitory Activity of Sinobambusa tootsik (Sieb.) Makino leaves
}

\author{
Xiao-Lin Qiu ${ }^{1}$, Qing-Feng Zhang ${ }^{\text {Corresp. 1, } 2}$ \\ ${ }^{1}$ College of New Energy and Environmental Engineering, Nanchang Institute of Technology, Nanchang, China \\ 2 Jiangxi Key Laboratory of Natural Product and Functional Food, College of Food Science and Engineering, Jiangxi Agricultural University, Nanchang, China \\ Corresponding Author: Qing-Feng Zhang \\ Email address: zhqf619@126.com
}

Background. Sinobambusa tootsik (Sieb.) Makino (S. tootsik) is one species of bamboo distributed in China, Japan and Vietnam. The chemical profile of its leaves and its potential application was unknown yet.

Methods. The chemical profile of S. tootsik was studied by HPLC and UPLC-DAD-QTOF-MS. The S. tootsik extract was prepared by extraction with $50 \%$ aqueous ethanol, followed by $\mathrm{H} 103$ macroporous resins adsorption and desorption processes. Pancreatic lipase inhibitory activity was determined using $p$ nitrophenyl palmitate as the substance, which was hydrolyzed by lipase to form coloured $p$-nitrophenol.

Results. Eighteen compounds were identified in $S$. tootsik. Most of them were the $C$-glycosylated derivatives of luteolin and apigenin, such as isoorientin, isoorientin-2"-O-rhamnoside and isovitexin. Isoorientin-2"-O-rhamnoside was the most dominant flavonoid in the sample. S. tootsik extract was prepared through resin adsorption/desorption with yield of $1.12 \pm 015 \%$ and total flavonoids content of $82 \pm 2 \mathrm{mg} / \mathrm{g}$ (in term of isoorientin). The extract exhibited pancreatic lipase inhibitory activity with IC50 value of $0.93 \mathrm{mg} / \mathrm{mL}$.

Conclusion. The chemical profile of $S$. tootsik leaves was uncovered for the first time. C-glycosyl flavonoids were the main constituents in the plant. The extract exhibited pancreatic lipase inhibitory activity and may have potential to be used as a food supplement for obesity controlling. 
1

2

3

4

5

6

7

8

9

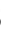

\section{Chemical Profile and Pancreatic Lipase Inhibitory Activity of Sinobambusa tootsik (Sieb.) Makino leaves}

Xiao-Lin Qiu ${ }^{1}$ and Qing-Feng Zhang ${ }^{1,2 *}$

${ }^{1}$ Nanchang Institute of Technology, College of New Energy and Environmental Engineering, Nanchang, China

92 Jiangxi Key Laboratory of Natural Product and Functional Food, College of Food Science and Engineering, Jiangxi Agricultural University, Nanchang, China

1 *Corresponding author: zhqf619@126.com, Phone/fax: 86-791-83813863. 


\section{ABSTRACT}

14 Background. Sinobambusa tootsik (Sieb.) Makino (S. tootsik) is one species of bamboo

15 distributed in China, Japan and Vietnam. The chemical profile of its leaves and its potential 16 application was unknown yet.

17 Methods. The chemical profile of $S$. tootsik was studied by HPLC and UPLC-DAD-QTOF-MS.

18 The $S$. tootsik extract was prepared by extraction with $50 \%$ aqueous ethanol, followed by $\mathrm{H} 103$ macroporous resins adsorption and desorption processes. Pancreatic lipase inhibitory activity was determined using $p$-nitrophenyl palmitate as the substance, which was hydrolyzed by lipase to form coloured $p$-nitrophenol.

Results. Eighteen compounds were identified in $S$. tootsik. Most of them were the $C$ 23 glycosylated derivatives of luteolin and apigenin, such as isoorientin, isoorientin-2"-Orhamnoside and isovitexin. Isoorientin-2"-O-rhamnoside was the most dominant flavonoid in the sample. S. tootsik extract was prepared through resin adsorption/desorption with yield of $1.12 \pm 015 \%$ and total flavonoids content of $82 \pm 2 \mathrm{mg} / \mathrm{g}$ (in term of isoorientin). The extract exhibited pancreatic lipase inhibitory activity with IC50 value of $0.93 \mathrm{mg} / \mathrm{mL}$.

Conclusion. The chemical profile of $S$. tootsik leaves was uncovered for the first time. $C$ glycosyl flavonoids were the main constituents in the plant. The extract exhibited pancreatic lipase inhibitory activity and may have potential to be used as a food supplement for obesity controlling. 
32 Keywords: Sinobambusa tootsik (Sieb.) Makino; chemical profile; $C$-glycosyl flavonoids;

33 pancreatic lipase; inhibition

34 


\section{INTRODUCTION}

36 Bamboo is a valuable plant distributed all over the world with more than 1500 species. The

37 bamboo shoots of some species, e.g. Phyllostachys heterocycla cv. pubescens (P. heterocycla),

38 were eaten as vegetable, while the leaves were used as herbal material in China. The flavonoids

39 extract of some bamboo species (Phyllostachys genus) were approved as the food antioxidant

40 and food resources in China (Wang et al., 2012). The pharmacological activities of bamboo

41 leaves were arise from the presence of phytochemicals. For instance, five $C$-Glycosyl flavones

42 were isolated from Fargesia robusta (Van Hoyweghen et al., 2010). Three chlorogenic acid

43 derivatives were isolated from Phyllostachys edulis and the antioxidant activity was evaluated

44 (Kweon et al., 2001). Wang et al. isolated three isoorientin derivatives from Bambusa. textilis

45 McClure (Wang et al., 2012). Previous, we identified twelve compounds in the leaves of

46 Bambusa multiplex cv. Fernleaf (B. multiplex), and found that $C$-glycosyl flavonoids including

47 vitexin, isovitexin, isoorientin and its derivatives, are the main chemical constitutes of the plant

48 (Qiu \& Zhang, 2019). Sinobambusa tootsik (Sieb.) Makino (S. tootsik) is one species of bamboo

49 distributed in China, Japan and Vietnam. To the best of our knowledge, the chemical profile of

50 its leaves has not been studied yet. To further uncover its potential application, the chemical

51 composition of $S$. tootsik was studied by HPLC and UPLC-QTOF-MS in the present study.

52 Furthermore, the pancreatic lipase inhibition activity of its extract was studied.

\section{MATERIALS AND METHODS}

\section{Chemicals and Plant materials}


55 Leaves of $S$. tootsik were collected in Jiangxi Agricultural University (with east longitude of

$56115^{\circ} 50^{\prime}$ and northern latitude of $28^{\circ} 46^{\prime}$ ) on Mar. 2019. The plant material was authenticated

57 based on morphological characters by Prof. Qing-Pei Yang (Jiangxi Agricultural University),

58 and the voucher specimen was deposited in Jiangxi Key Laboratory of Natural Product and

59 Functional Food. The leaves was dried at $60{ }^{\circ} \mathrm{C}$ and smashed to filter through 40 mesh sieve.

60 Isoorientin standard (>98\%) was purchased from Beijing Solarbio Science \& Technology Co.,

61 Ltd (Beijing, China). HPLC grade acetonitrile was purchased from Anhui Tedia High Purity

62 Solvents Co., Ltd (Anqin, China). Porcine pancreatic lipase (extract powder, 15-35 units/mg,

63 catalog number L111237) was purchased from Aladdin Chemistry Co. Ltd. (Shanghai, China;

64 http://www.aladdin-e.com). Milli-Q water was used throughout the study. All other reagents

65 used were analytical grade.

\section{Sample extraction}

67 A $0.1 \mathrm{~g}$ aliquot of $S$. tootsik powder was mixed with $5.0 \mathrm{~mL}$ of $50 \%$ aqueous ethanol. After sonicating for $30 \mathrm{~min}$ in a bath sonicator $(100 \mathrm{~W}, 45 \mathrm{kHz}$, Kunshan, China), the mixture was centrifuged at $1100 \mathrm{~g}$ for $5 \mathrm{~min}$. The supernatant was filtered by $0.22 \mathrm{~mm}$ pore size filter and then used for HPLC and UPLC-DAD-QTOF-MS analysis.

71 For $S$. tootsik extract preparation, $50 \mathrm{~g}$ of $S$. tootsik sample was extracted for twice with $500 \mathrm{~mL}$ 72 of $50 \%$ aqueous ethanol each time. After centrifugation at $1100 \mathrm{~g}$ for $5 \mathrm{~min}$, the supernatant was 73 combined together. The extract was condensed to about $500 \mathrm{~mL}$ by vacuum rotavapor at $50 \circ \mathrm{C}$.

74 The concentrates was two times diluted by water. Then, the extract was pumped through a fixed 
75 bed of H103 macroporous resin with diameter of $1.5 \mathrm{~cm}$ and height of $40 \mathrm{~cm}$ in a glass column.

76 The flow rate was $10 \mathrm{~mL} / \mathrm{min}$. After adsorption, the fixed bed was desorbed with $4 \mathrm{BV}$ of $90 \%$

77 ethanol with flow rate of $5 \mathrm{~mL} / \mathrm{min}$. The eluent was concentrated by vacuum rotavapor at $50{ }^{\circ} \mathrm{C}$

78 and then lyophilized to obtain the extract.

\section{UPLC-DAD-QTOF-MS analysis}

80 The chemical identification was performed on a QTOF 5600-plus mass spectrometer equipped

81 with Turbo V sources and a Turbolonspray interface (AB Sciex Corporation, Foster City, CA,

82 USA) coupled to a Shimadzu LC-30A UPLC-DAD system (Shimadzu Corporation, Kyoto,

83 Japan). Acquity UPLC BEH C18 column $(2.1 \mathrm{~mm} \times 100 \mathrm{~mm}, 1.7 \mu \mathrm{m}$, Waters $)$ was used. The

84 flow rate was $0.3 \mathrm{~mL} / \mathrm{min}$ with injection volume of $1 \mu \mathrm{L}$ and column temperature of $40{ }^{\circ} \mathrm{C}$. The

85 mobile phase was acetonitrile (A) and $0.1 \%$ formic acid aqueous solution (B) using a linear

86 gradient program of $0-30 \mathrm{~min}, 5-40 \%(\mathrm{~A})$. The mass spectrometer was operated in the negative

87 ion mode. Ultrapure nitrogen was used as the ion source gas 1 (50 psi), ion source gas 2 (50 psi),

88 and curtain gas (40 psi). The Turbo Ion Spray voltage and temperature were set at $-4500 \mathrm{~V}$ and

$89500{ }^{\circ} \mathrm{C}$, respectively. Declustering potential, collision energy, and collision energy spread were

90 set at $100 \mathrm{~V},-40 \mathrm{~V}$, and $10 \mathrm{~V}$, respectively. Data acquisition was performed with Analyst 1.6

91 software (AB Sciex).

\section{HPLC quantification Analysis}

93 The HPLC Analysis was performed on an Agilent 1260 HPLC system equipped with an 
94 autosampler and DAD detector. A Symmetry C18 column $(250 \mathrm{~mm} \times 4.6 \mathrm{~mm}$ i.d., $5 \mu \mathrm{m}$; Waters,

95 USA) was used as the stationary phase. The mobile phase consisted of acetonitrile (A) and $0.1 \%$

96 acetic acid aqueous solution (B). The flow rate was $1 \mathrm{~mL} / \mathrm{min}$ with linear gradient program of 0-

$9730 \mathrm{~min}, 1-40 \% \mathrm{~A} ; 30-35 \mathrm{~min}, 40 \%$ A. Detected wavelength was $349 \mathrm{~nm}$ with injection volume

98 of $10 \mu \mathrm{L}$ and column temperature of $40^{\circ} \mathrm{C}$.

111 Lipase inhibition activity $(\%)=\frac{\mathrm{V}_{\mathrm{b}}-\mathrm{V}_{\mathrm{s}}}{\mathrm{V}_{\mathrm{b}}} \times 100$

112 Where $\mathrm{V}_{\mathrm{b}}$ and $\mathrm{V}_{\mathrm{s}}$ were the enzyme activity in the absence and presence of $S$. tootsik extract, 
113 respectively. Orlistat was used as the positive control. The same reaction mixture but without

114 lipase was used as the negative control, in which no absorbance change was found.

\section{Fluorescence quench measurements}

116 A $1.0 \mathrm{~mL}$ aliquot of the lipase solution was mixed with $4 \mathrm{~mL}$ of Tris-buffer. Subsequently, 0,5 ,

$11710,15,20,25$ and $30 \mu \mathrm{L}$ of $S$. tootsik extract $(10 \mathrm{mg} / \mathrm{mL}$ in $50 \%$ aqueous ethanol) was added, 118 respectively. The fluorescence spectra of the mixture was recorded between 300 to $450 \mathrm{~nm}$ under

119 the excitation wavelength of $280 \mathrm{~nm}$. A 970 CRT spectrofluophotometer (Shanghai Scientific 120 Instruments Limited Company, Shanghai, China) was used, and the excitation and emission 121 bandwidths were set at $10 \mathrm{~nm}$.

\section{Statistical Analysis}

123 Data were expressed as the mean \pm standard deviation (SD) of triplicates. Statistical analysis, 124 plotting, and curve fitting were performed by Origin 7.0 (Origin Lab Co., Northampton, MA, 125 USA).

RESULT AND DISCUSSION

\section{Chemical profile of $S$. tootsik}

128 Figure 1 corresponds to the chromatograms of $S$. tootsik detected by QTOF-MS and DAD after 129 UPLC separation. By the QTOF-MS detector, the molecular mass of each peak and its further $130 \mathrm{MS}^{2}$ spectrum was obtained. The chemical identification was accomplished by comparing these 
131 information with published literature. The details were listed in Table 1 . A total of 18

132 components were identified. Most of them were the $C$-glycosylated derivatives of luteolin and

133 apigenin, such as isoorientin, isoorientin-2"-O-rhamnoside and isovitexin. Besides, some other

$134 \mathrm{C}$-glycosyl and $\mathrm{O}$-glycosyl flavonoids were found, such as isoscoparin- $O$-deoxyhexoside and

135 kaempferol-O-glucoside. Two non-flavonoid compounds, feruloylquinic acid and roseoside,

136 were also found. S. tootsik belongs to the family of Poaceae. Many studied showed that the main

137 secondary metabolite found in the leaves of Poaceae plants were $C$-glycosyl flavonoids, for

138 instance, barley, maize, wheat, rice, etc (Brazier-Hicks et al., 2009, Ferreres et al., 2008).

139 Previously, we have studied the chemical constituents in the leaves of $B$. multiplex, another

140 bamboo species (Qiu \& Zhang, 2019). It was found that $C$-glycosylated derivatives of luteolin

141 and apigenin were the main components of both species. However, the specific flavonoids

142 between the two plants were different. Only apigenin 6- $C$-pentoside-8- $C$-glucoside, isoorientin

143 and isovitexin were found in the both species.

Figure 2A was the HPLC chromatogram of $S$. tootsik detected at $349 \mathrm{~nm}$. With the results of

UPLC-DAD-Q-TOF-MS analysis (Figure 1), the five main peaks in the HPLC chromatogram were identified. The peak of isoorientin (peak 2) was further validated by comparing the retention time and UV spectra with standard marker. Form the peak area, it was found that isoorientin-2"-O-rhamnoside (peak 1) was the most dominant flavonoid in $S$. tootsik, followed by isoscoparin- $O$-deoxyhexoside (peak 4) and apigenin 6-C-[2"-O-deoxyhexoside]-pentoside (peak 
151

152

153

154

155

156

157

158

159

160

161

162

163

164

165

166

167

168

169

flavonoid, followed by isovitexin (Qiu \& Zhang, 2019). The UV spectra of the five peaks were

presented in Figure 2B. It was found that the UV spectra of peaks 1,2 and 4 were very similar with maxmium absorption around $348 \mathrm{~nm}$, while the maxmium absorption of peaks 3 and 5 was around $338 \mathrm{~nm}$.

\section{S. tootsik extract preparation}

Through 50\% aqueous ethanol extraction, followed by H103 macroporous resins adsorption and desorption processes, the yield of $S$. tootsik extract was $1.12 \pm 015 \%$. HPLC analysis showed that the chemical profile was unchanged (Figure 2). However, the chemical content reflected by peak area were about 29.8 times increased. Besides isoorientin, most of the other flavonoids identified in $S$. tootsik were market unavailable. The calibration curves of isoorientin were $\mathrm{Y}=24.82 \mathrm{X}$, with correlation coefficient of 0.999 , where $\mathrm{Y}$ was the peak area and $\mathrm{X}$ was concentration of isoorientin $(5-200 \mu \mathrm{g} / \mathrm{ml})$. By submitted the area sum of peak 1 to 5 to the calibration curves, the total flavonoids content in $S$. tootsik extract was calculated as $82 \pm 2 \mathrm{mg} / \mathrm{g}$ in term of isoorientin.

\section{Pancreatic lipase inhibitory activity of $S$. tootsik extract}

Obesity is becoming one of the biggest threats to human health around the world. Before being absorbed by the small intestine, food fats need first be hydrolyzed by lipase into monoglycerol and free fatty acids. Thus, the inhibition of lipase, especially pancreatic lipase, could effectively reduce the absorption of fat in the diet, thereby controlling and treating obesity (Bialecka-Florjanczyk et al., 2018; Birari \& Bhutani, 2007; Buchholz \& Melzig, 2015; Yun, 
170 2010). Thus, the finding of lipase inhibitor from natural source is getting more and more

171 attention. Many flavonoids from plant source show pancreatic lipase inhibitory activity, such as

172 luteolin, genistein, hyperin, kaempferol, etc (Buchholz \& Melzig, 2015). Lee et al. found that the

$173 C$-glycosylated derivatives of luteolin on A-ring exhibited much stronger pancreatic lipase

174 inhibitory activity than luteolin (Lee et al., 2010). The main identified flavonoids in S. tootsik

175 were the $C$-glycosylated derivatives of luteolin and apigenin. Thus, the pancreatic lipase

176 inhibitory activity of $S$. tootsik extract was studied in the present study. The result showed that

177 the pancreatic lipase inhibitory activity of $S$. tootsik extract steadily increased with the

178 concentration, and the IC50 value was about $0.93 \mathrm{mg} / \mathrm{mL}$ (Figure 3 ). In comparison, the IC50

179 value of Orlistat, the clinically approved pancreatic lipase inhibitor, was $74 \mathrm{ng} / \mathrm{mL}$. Many plant

extracts with components of saponins, phenolic acids, and/or flavonoids, possess pancreatic

lipase inhibitory effects (Buchholz \& Melzig, 2015; de la Garza et al., 2011). For instance,

Crocus cancellatus subsp. damascenus extract with main constituents of catechin, ferulic and

caffeic acids, induced $50.39 \%$ of inhibition of lipase activity at $5 \mathrm{mg} / \mathrm{mL}$ (Loizzo et al., 2016).

The acetic extracts of Aronia melanocarpa L. and its cyanidin-3-glucoside fraction exhibited

pancreatic lipase inhibitory activities with IC50 values of 83.45 and $1.74 \mathrm{mg} / \mathrm{mL}$, respectively

(Worsztynowicz et al., 2014). The IC50 value of Moricandia arvensis (L.) DC methanolic

extract with main constituents of flavonoid glycosides was $2.06 \mathrm{mg} / \mathrm{mL}$, while the IC50 value of

Orlistat was $0.018 \mathrm{mg} / \mathrm{mL}$ in the study (Marrelli et al., 2018).

Fluorometric analysis showed that the addition of $S$. tootsik extract could gradually quench the 
190

191

192

193

194

195

endogenous fluorescence of pancreatic lipase (Figure 4). Furthermore, it also caused the red shift of maximum emission wavelength. These phenomenons implied that the flavonoids in S. tootsik extract could bind on the enzyme. If the ligand is a monomeric compound, the fluorescence titration results can be further used to calculated the binding constant and binding site of the complexes using Stern-Volmer equations. However, in the present study, the $S$. tootsik extract is a mixture without definite molecular weight, and Stern-Volmer equations can't be applied.

Although the lipase inhibitory activity of $S$. tootsik extract was far weaker than Orlistat, as an abundant and safe natural product, it may also have potential to be used as a food supplement for obesity controlling. The in vivo study of its anti-obesity is in progress in our Lab.

\section{CONCLUSION}

The chemical profile of $S$. tootsik was studied by HPLC and UPLC-DAD-QTOF-MS. Eighteen compounds were identified, most of them were the $C$-glycosylated derivatives of luteolin and apigenin, such as isoorientin, isoorientin-2"-O-rhamnoside and isovitexin. Isoorientin-2"-Orhamnoside was the most dominant flavonoid in the sample. S. tootsik extract was prepared through resin adsorption/desorption with yield of $1.12 \pm 0.15 \%$ and total flavonoids content of $82 \pm 2 \mathrm{mg} / \mathrm{g}$ (in term of isoorientin). The extract exhibited pancreatic lipase inhibitory activity with IC50 value of about $0.93 \mathrm{mg} / \mathrm{mL}$.

\section{Funding}

This work was supported by the National Natural Science Foundation of China (Grant Number

Peer) reviewing PDF | (2019:06:38752:2:0:NEW 19 Aug 2019) 
209

210

211

212

213

214

215

216

217

218

219

220

221

222

223

224

225

226

227

31760461).

\section{Competing Interests}

The authors declare there are no competing interests

\section{REFERENCES}

Bezerra AG, Negri G, Duarte-Almeida JM, Smaili SS, Carlini EA. 2016. Phytochemical analysis of hydroethanolic extract of Turnera diffusa Willd and evaluation of its effects on astrocyte cell death. Einstein (São Paulo) 14(1):56-63.

Bialecka-Florjanczyk E, Fabiszewska AU, Krzyczkowska J, Kurylowicz A. 2018. Synthetic and natural lipase inhibitors. Mini Reviews in Medicinal Chemistry 18(8):672-683.

Birari RB, Bhutani KK. 2007. Pancreatic lipase inhibitors from natural sources: unexplored potential. Drug Discovery Today 12(19-20):879-889.

Brazier-Hicks M, Evans KM, Gershater MC, Puschmann H, Steel PG, Edwards R. 2009. The C-glycosylation of flavonoids in cereals. Journal of Biological Chemistry 284:1792617934.

Buchholz T, Melzig MF. 2015. Polyphenolic compounds as pancreatic lipase inhibitors. Planta Medica 81(10): 771-783.

de la Garza AL, Milagro FI, Boque N, Campión J, Martínez JA. 2011. Natural inhibitors of pancreatic lipase as new players in obesity treatment. Planta Medica 77(08):773-785

Ferreres F, Andrade PB, Valentão P, Gil-Izquierdo A. 2008. Further knowledge on barley 
(Hordeum vulgare L.) leaves $O$-glycosyl- $C$-glycosyl flavones by liquid chromatographyUV diode-array detection-electrospray ionisation mass spectrometry. Journal of Chromatography A 1182(1):56-64.

Figueirinha A, Paranhos A, Pérez-Alonso JJ, Santos-Buelga C, Batista MT. 2008. Cymbopogon citratus leaves: Characterization of flavonoids by HPLC-PDA-ESI/MS/MS and an approach to their potential as a source of bioactive polyphenols. Food Chemistry 110(3):718-728

Han J, Ye M, Xu M, Sun J, Wang B, Guo D. 2007. Characterization of flavonoids in the traditional Chinese herbal medicine-Huangqin by liquid chromatography coupled with electrospray ionization mass spectrometry. Journal of Chromatography B 848(2):355-362.

Ibrahim RM, El-Halawany AM, Saleh DO, El Naggar EMB, El-Shabrawy AERO, ElHawary SS. 2015. HPLC-DAD-MS/MS profiling of phenolics from Securigera securidaca flowers and its anti-hyperglycemic and anti-hyperlipidemic activities. Revista Brasileira de Farmacognosia 25(2):134-141.

Iswaldi I, Arráez-Román D, Rodríguez-Medina I, Beltrán-Debón R, Joven J, SeguraCarretero A, Fernández-Gutiérrez A. 2011. Identification of phenolic compounds in aqueous and ethanolic rooibos extracts (Aspalathus linearis) by HPLC-ESI-MS (TOF/IT). Analytical and Bioanalytical Chemistry 400(10):3643-3654.

Kweon MH, Hwang HJ, Sung HC. 2001. Identification and antioxidant activity of novel chlorogenic acid derivatives from bamboo (Phyllostachys edulis). Journal of Agricultural and Food Chemistry 49(10):4646-4655. 
249 Lee EM, Lee SS, Chung BY, Cho JY, Lee IC, Ahn SR, Jang SJ, Kim TH. 2010. Pancreatic

250 lipase inhibition by $C$-glycosidic flavones isolated from Eremochloa ophiuroides. 251 Molecules 15(11):8251-8259.

252

253

254

255

Loizzo MR, Marrelli M, Pugliese A, Conforti F, Nadjafi F, Menichini F, Tundis R. 2016. Crocus cancellatus subsp. damascenus stigmas: chemical profile, and inhibition of $\alpha-$ amylase, $\alpha$-glucosidase and lipase, key enzymes related to type 2 diabetes and obesity. Journal of Enzyme Inhibition and Medicinal Chemistry 31(2):212-218.

Marrelli M, Morrone F, Argentieri M, Gambacorta L, Conforti F, Avato P. 2018. Phytochemical and Biological Profile of Moricandia arvensis (L.) DC.: An Inhibitor of Pancreatic Lipase. Molecules 23(11):2829.

Worsztynowicz P, Napierala M, Białas W, Grajek W, Olkowicz M. 2014. Pancreatic $\alpha$ amylase and lipase inhibitory activity of polyphenolic compounds present in the extract of black chokeberry (Aronia melanocarpa L.). Process Biochemistry 49(9), 1457-1463.

Ozarowski M, Piasecka A, Paszel-Jaworska A, Chaves DSDA, Romaniuk A, Rybczynska M, Gryszczynskab A, Sawikowskag A, Kachlickic P, Mikolajczak PL, SeremakMrozikiewicz A, Klejewski A, Seremak-Mrozikiewicz A. 2018. Comparison of bioactive compounds content in leaf extracts of Passiflora incarnata, P. caerulea and P. alata and in vitro cytotoxic potential on leukemia cell lines. Revista Brasileira de Farmacognosia 28(2):179-191.

Qiu XL, Guo YX, Zhang QF. 2018. Chemical profile and antioxidant activity of Gynura bicolor DC. ethanolic extract. International Journal of Food Properties 21(1):407-415. 
270 Qiu XL, Zhang QF. 2019. Identification and quantification of main flavonoids in the leaves of

271

272

273

274

275

276

277

278

279

280

281

282

283

284

285

286

Bambusa multiplex cv. Fernleaf. Natural Product Research

DOI:10.1080/14786419.2019.1569013.

Singh AP, Wilson T, Luthria D, Freeman MR, Scott RM, Bilenker D, Shah S, Somasundaram S, Vorsa N. 2011. LC-MS-MS characterisation of curry leaf flavonols and antioxidant activity. Food Chemistry 127(1):80-85.

Spínola V, Pinto J, Castilho PC. 2015. Identification and quantification of phenolic compounds of selected fruits from Madeira Island by HPLC-DAD-ESI-MS ${ }^{\mathrm{n}}$ and screening for their antioxidant activity. Food Chemistry 173:14-30.

Van Hoyweghen L, Karalic I, Van Calenbergh S, Deforce D, Heyerick A. 2010. Antioxidant flavone glycosides from the leaves of Fargesia robusta. Journal of Natural Products 73(9):1573-1577

Wang J, Yue YD, Tang F, Sun J. 2012. TLC screening for antioxidant activity of extracts from fifteen bamboo species and identification of antioxidant flavone glycosides from leaves of Bambusa. textilis McClure. Molecules 17(10):12297-12311.

Yun JW. 2010. Possible anti-obesity therapeutics from nature-A review. Phytochemistry 71(1415):1625-1641. 


\section{Table 1 (on next page)}

Mass characterizations of main peak in the chromatogram of Sinobambusa tootsik (Sieb.) Makino by UPLC-QTOF-MS 


\begin{tabular}{|c|c|c|c|c|c|}
\hline $\begin{array}{l}\text { Peak } \\
\text { No. }\end{array}$ & $\begin{array}{l}\text { RT } \\
(\min )\end{array}$ & $\begin{array}{c}{[\mathrm{M}-\mathrm{H}]^{-}} \\
(\mathrm{m} / \mathrm{z})\end{array}$ & Fragment ions $(\mathrm{m} / \mathrm{z})$ & Proposed structure & Reference \\
\hline 1 & 6.10 & 367.1033 & 193(45), 134(100), 117(10) & Feruloylquinic acid & Qiu et al., 2018 \\
\hline 2 & 6.97 & 609.1449 & 519(25), 489(60), 399(70), 369(100) & Quercetin-3-O-robinobioside & Iswaldi et al., 2011 \\
\hline 3 & 7.60 & 431.1915 & $385(15), 205(35), 153(100)$ & Roseoside & Spínola et al., 2015 \\
\hline 4 & 7.78 & 519.1707 & $325(10), 265(33), 223(60), 205(100), 190(55)$ & Unidentified & \\
\hline 5 & 8.09 & 489.159 & 223(40), 205(100),190(80), 164(27) & Unidentified & \\
\hline 6 & 8.69 & 371.098 & 121(100), 249(45), 231(10) & Unidentified & \\
\hline \multirow{4}{*}{7} & & & & Apigenin 6- $C$-pentoside- & \\
\hline & 9.14 & 563.1393 & $353(100), 383(65), 443(45), 473(32)$ & & Ozarowski et al., 2018 \\
\hline & & & & 8 - $C$-glucoside & \\
\hline & 9.14 & 447.0924 & $327(100), 357(70), 297(55), 285(35)$ & Isoorientin (luteolin 6- $C$-glucoside) & Figueirinha et al., 2008 \\
\hline 8 & 9.26 & 593.1504 & $\begin{array}{c}298(100), 473(85), 327(55), 309(40), 357(35), \\
429(25)\end{array}$ & Isoorientin-2"-O-rhamnoside & Ibrahim et al., 2015 \\
\hline 9 & 9.78 & 613.213 & $181(100), 387(85), 166(30), 205(25), 399(20)$ & Unidentified & \\
\hline \multirow[t]{3}{*}{10} & 10.68 & 533.128 & $\begin{array}{c}353(100), 383(90), 443(50), 473(40), 365(25) \\
297(23)\end{array}$ & Apigenin 6,8 -di- $C$-pentoside & Ozarowski et al., 2018 \\
\hline & 10.68 & 577.1546 & 293(100), 413(35), 323(15), 311(15), 457(10), & Isovitexin-2"-O-rhamnoside & Ibrahim et al., 2015 \\
\hline & 10.71 & 431.0986 & $311(100), 341(35), 283(75)$ & Isovitexin (apigenin 6-C-glucoside) & Ibrahim et al., 2015 \\
\hline \multirow[t]{3}{*}{11} & 11.27 & 607.1649 & $323(100), 443(40), 308(20), 341(15)$ & Isoscoparin- $O$-deoxyhexoside & Ozarowski et al., 2018 \\
\hline & 11.29 & 447.091 & $285(100))$ & Kaempferol- $O$-glucoside & Singh et al., 2011 \\
\hline & & & & Chrysin 6- $C$-deoxyhexoside- & \\
\hline \multirow[t]{3}{*}{12} & 12.89 & 561.1595 & $561(100), 457(30), 399(14), 337(18), 295(40)$ & & Ozarowski et al., 2018 \\
\hline & & & & 7-O-glucoside & \\
\hline & 13.01 & 637.1759 & $329(100), 314(15), 299(10)$ & 3,4 -Dihydroxy-5,6-dimethoxy & Han et al., 2007 \\
\hline
\end{tabular}




\begin{tabular}{|c|c|c|c|c|c|}
\hline & & & & -7-O-rutinoside flavone & \\
\hline 13 & 13.41 & 547.1446 & 293(100), 383(85),341(35), $311(28)$ & $\begin{array}{l}\text { Apigenin 6-C-[2"- } O \\
\text {-deoxyhexoside] - pentoside }\end{array}$ & Ozarowski et al., 2018 \\
\hline 14 & 13.69 & 577.1546 & $311(100), 415(50), 397(15)$ & $\begin{array}{l}\text { Apigenin-6-C-deoxyhexoside- } \\
\text { 7-O-glucoside }\end{array}$ & Ozarowski et al., 2018 \\
\hline 15 & 14.08 & 575.1392 & $\begin{array}{c}325(100), 297(100), 411(100), 337(70), 285(70) \\
367(55)\end{array}$ & $\begin{array}{l}\text { "X"-O-Rhamnosyl C-(6-deoxy } \\
\text {-pento-hexos-ulosyl) luteolin }\end{array}$ & Figueirinha et al., 2008 \\
\hline 16 & 14.45 & 577.1549 & $311(100), 298(70), 415(70), 473(50), 327(35)$ & $\begin{array}{l}\text { Apigenin-8- } C \text {-deoxyhexoside-7 } \\
\text {-O-glucoside }\end{array}$ & Ozarowski et al., 2018 \\
\hline 17 & 15.52 & 559.1441 & $\begin{array}{c}457(10), 395(95), 321(100), 309(25), 293(50) \\
281(30), 269(60)\end{array}$ & $\begin{array}{l}\text { Apigenin-8-C-[6-deoxy-2-O- } \\
\text { rhamnosyl]-xylo-Hexos-3-uloside }\end{array}$ & Bezerra et al., 2016 \\
\hline 18 & 16.00 & 589.1554 & $425(100), 351(65), 325(35), 299(35)$ & Unidentified & \\
\hline
\end{tabular}

2

3 
Figure 1

The chromatograms of $S$. tootsik detected by QTOF-MS (a) and DAD (b) after UPLC separation.

Line a: base peak chromatogram of QTOF-MS; Line b: detected at $349 \mathrm{~nm}$.

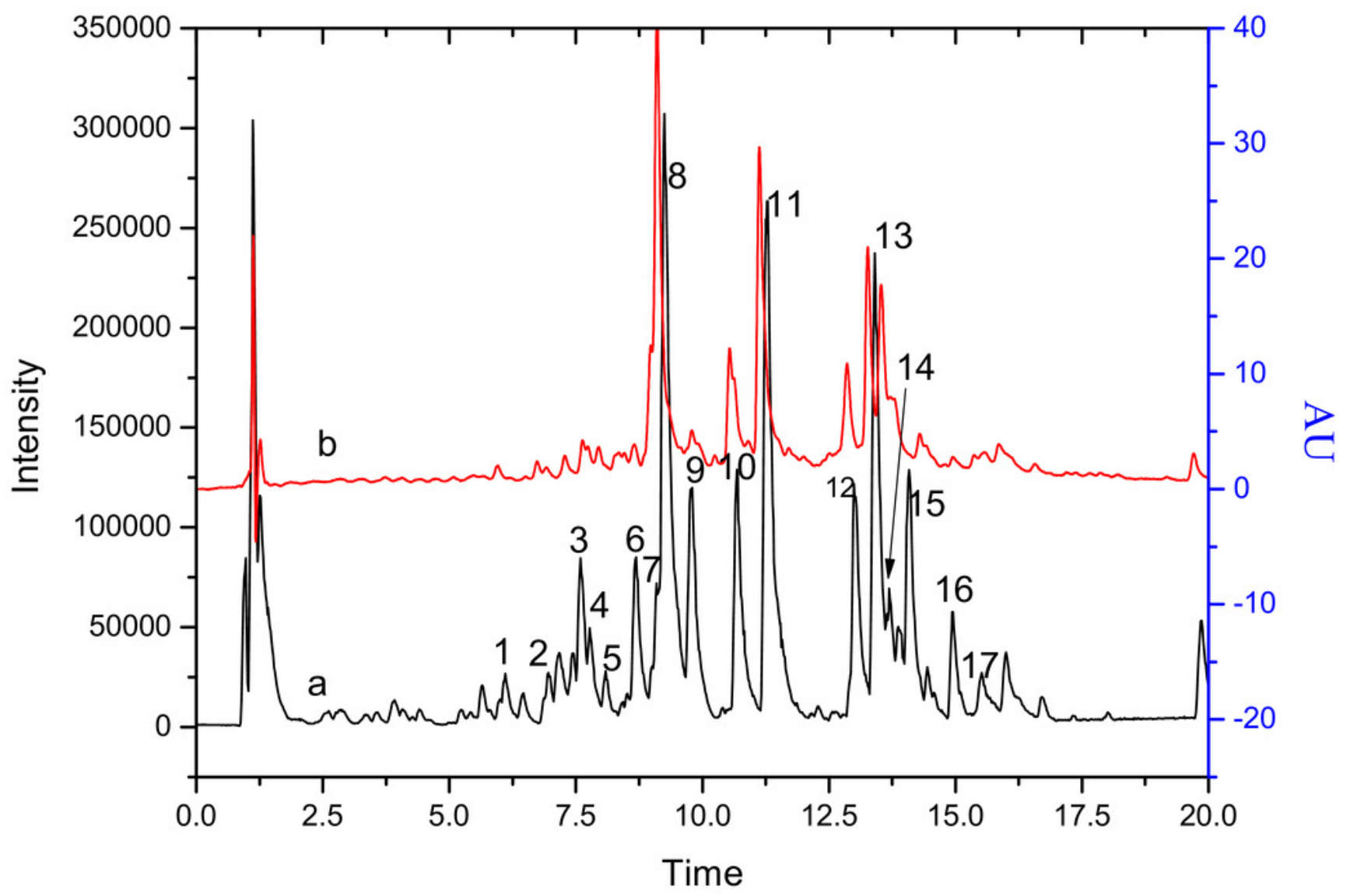


Figure 2

A: HPLC chromatogram of S. tootsik extract before (a) and after (b) resin purification; B: The UV spectra of peak 1-5.

Peaks: 1, Isoorientin-2"-O-rhamnoside; 2, Isoorientin; 3, Isovitexin-2"'-O-rhamnoside; 4, Isoscoparin-O-deoxyhexoside; 5, Apigenin 6-C-[2"-O-deoxyhexoside]-pentoside. 

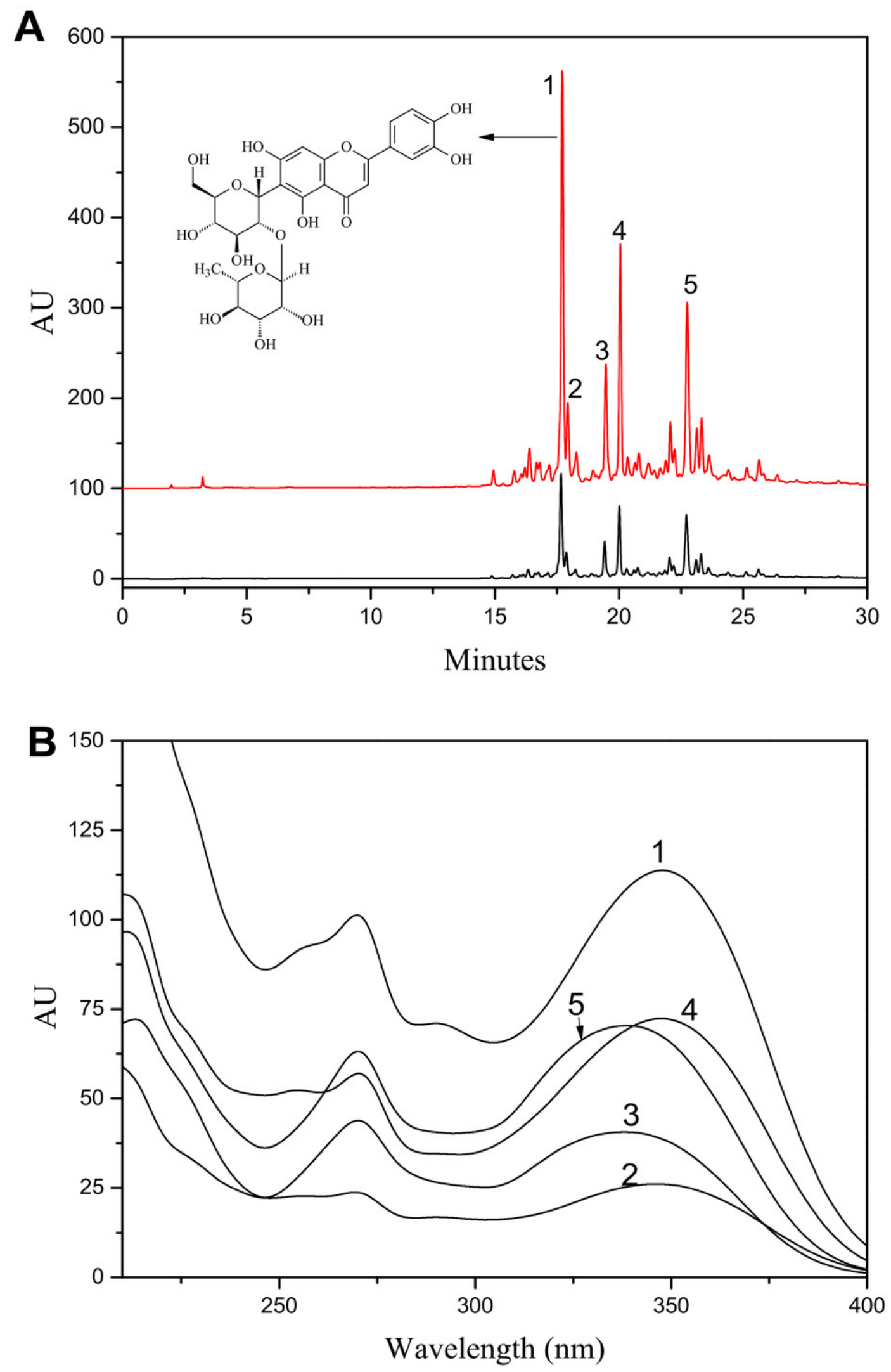
Figure 3

The lipase inhibitory activity of $S$. tootsik extract.

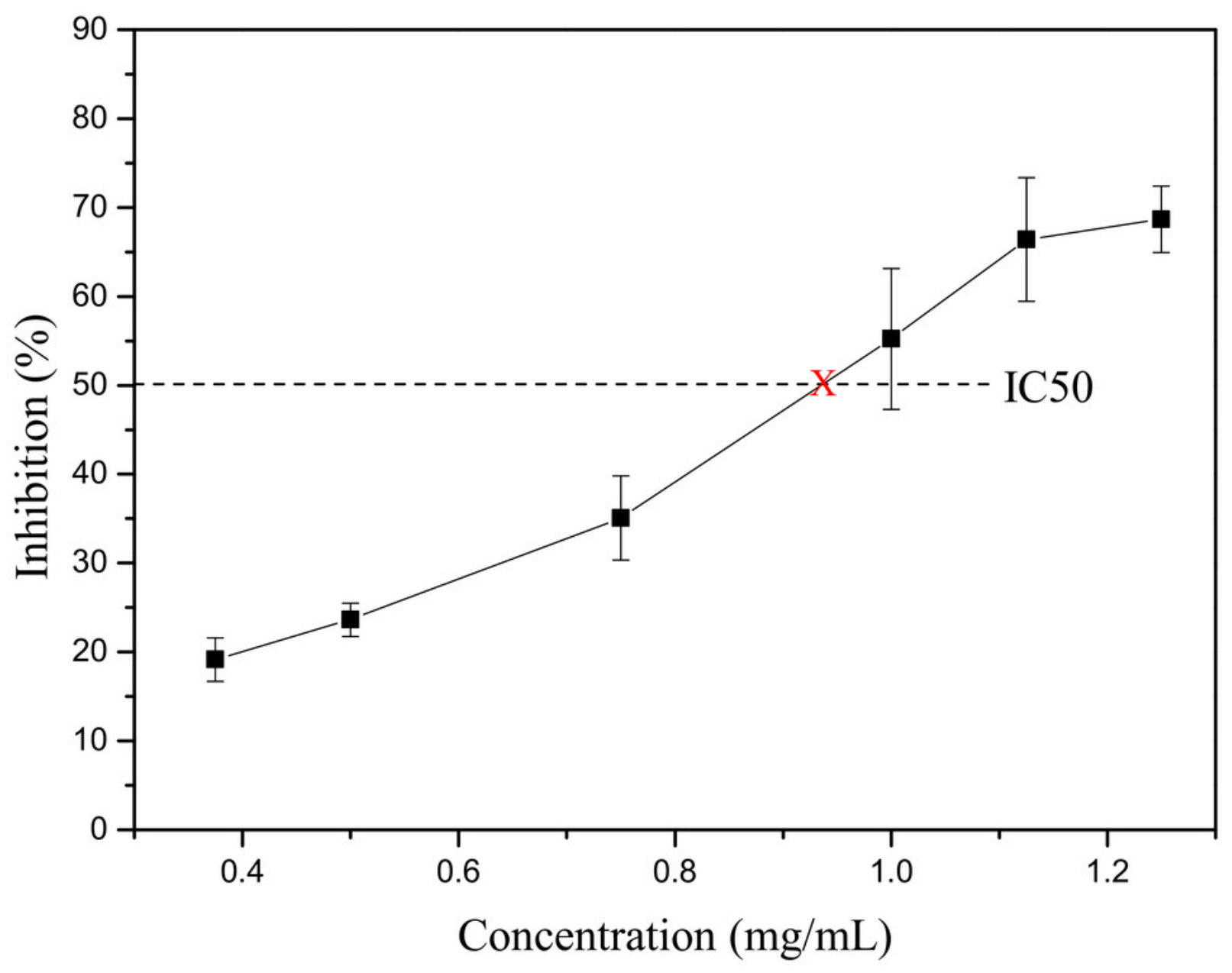


Figure 4

The effect of S. tootsik extract on fluorescence emission spectra of pancreatic lipase.

The concentrations of $S$. tootsik extract from 1-7 were $0,10,20,30,40,50,60 \mu \mathrm{g} / \mathrm{mL}$, respectively.

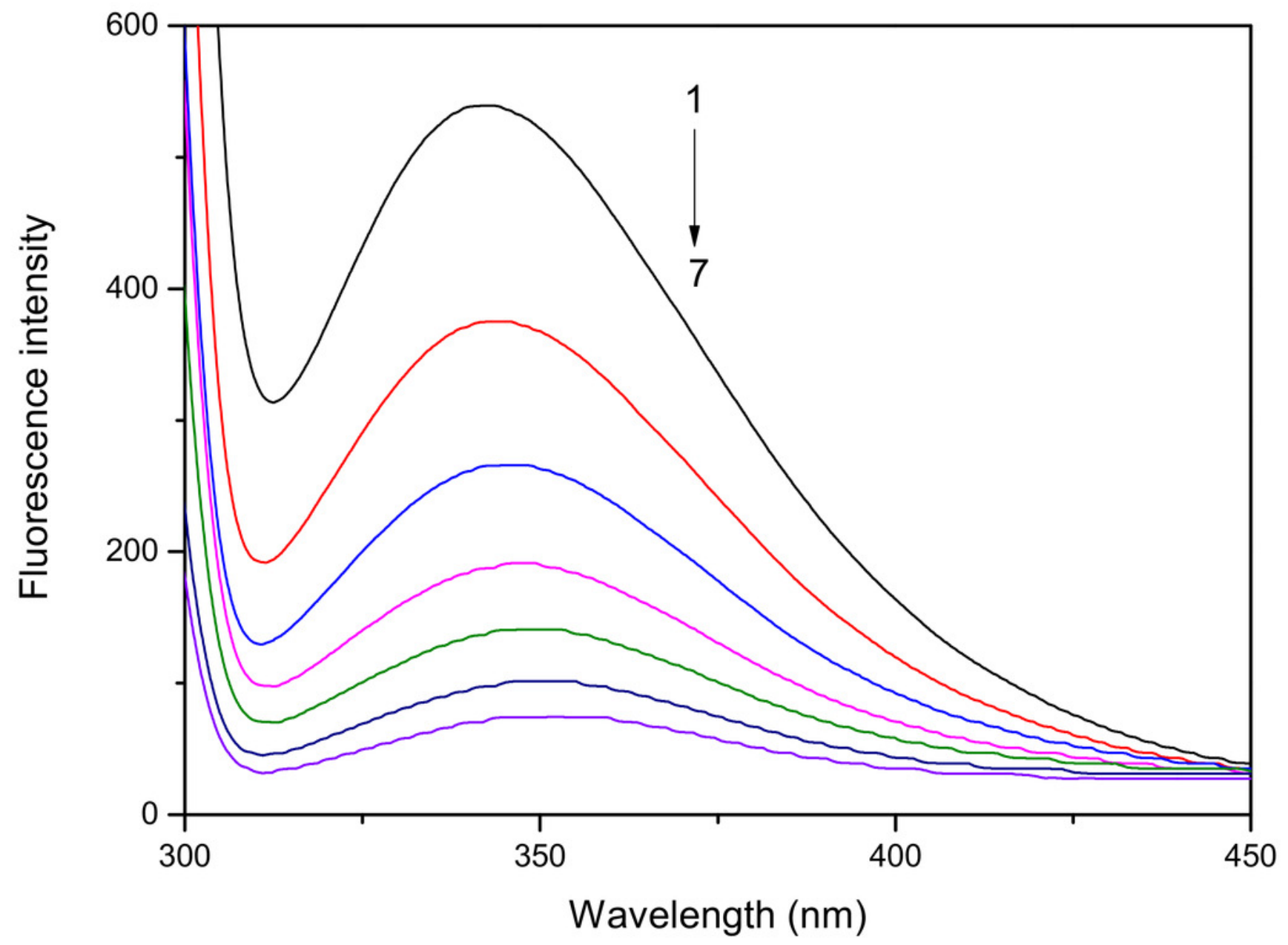

